\title{
Infodemic Pathways: Evaluating the Role That Traditional and Social Media Play in Cross-National Information Transfer
}

\section{OPEN ACCESS}

Edited by:

Sevasti Chatzopoulou, Roskilde University, Denmark

Reviewed by:

Joseph Downing,

London School of Economics and

Political Science, United Kingdom Jill Sheppard,

Australian National University, Australia

${ }^{*}$ Correspondence: Aengus Bridgman aengus.bridgman@mail.mcgill.ca

Specialty section:

This article was submitted to Political Participation,

a section of the journal Frontiers in Political Science

Received: 01 January 2021 Accepted: 01 March 2021 Published: 29 March 2021

Citation:

Bridgman A, Merkley E, Zhilin O, Loewen PJ, Owen T and Ruths D

(2021) Infodemic Pathways: Evaluating the Role That Traditional and Social Media Play in Cross-National Information Transfer. Front. Polit. Sci. 3:648646. doi: 10.3389/fpos.2021.648646

\begin{abstract}
Aengus Bridgman ${ }^{1 *}$, Eric Merkley ${ }^{2}$, Oleg Zhilin ${ }^{3}$, Peter John Loewen ${ }^{2}$, Taylor Owen ${ }^{4}$ and Derek Ruths ${ }^{3}$

${ }^{1}$ Department of Political Science, Mc Gill University, Montreal, QC, Canada, ${ }^{2}$ Department of Political Science, University of Toronto, Toronto, ON, Canada, ${ }^{3}$ Department of Computer Science, McGill University, Montreal, QC, Canada, ${ }^{4}$ Max Bell

School of Public Policy, McGill University, Montreal, QC, Canada
\end{abstract}

The COVID-19 pandemic has occurred alongside a worldwide infodemic where unprecedented levels of misinformation have contributed to widespread misconceptions about the novel coronavirus. Conspiracy theories, poorly sourced medical advice, and information trivializing the virus have ignored national borders and spread quickly. This information spread has occurred despite generally strong preferences for domestic national media and social media networks that tend to be geographically bounded. How, then, is (mis)information crossing borders so rapidly? Using social media and survey data, we evaluate the extent to which consumption and propagation patterns of domestic and international traditional news and social media can help inform theorizing about crossnational information spread. In a detailed case study of Canada, we employ a large multi-wave survey and a massive data set of Canadian Twitter users. We show that the majority of misinformation circulating on Twitter that is shared by Canadian accounts is retweeted from U.S.-based accounts. Moreover, exposure to U.S.-based media outlets is associated with COVID-19 misperceptions and increased exposure to U.S.-based information on Twitter is associated with an increased likelihood to post misinformation. We thus theorize and empirically identify a key globalizing infodemic pathway: disregard for national origin of social media posting.

Keywords: social media, infodemic, COVID-19, Canada, misinformation, Twitter, information ecosystem, news media

\section{INTRODUCTION}

The COVID-19 pandemic has been accompanied by a worldwide infodemic. Unprecedented levels of misinformation have contributed to widespread misconceptions about the novel coronavirus. Conspiracy theories, poorly sourced medical advice, and information trivializing the virus have ignored national borders, spreading rapidly and globally. This infodemic has the capacity to change important attitudes and behaviors that in turn can impact transmission patterns (Larson, 2018; Kim et al., 2019). Ultimately, it can change the scale and lethality of a pandemic. Infodemic information can come from a range of sources, but there is reason to be particularly concerned about the role played by social media, such as Facebook and Twitter. These platforms have been found to be a key pathway by which medically misleading information has entered into more mainstream discourse (Jang et al., 2019). 
There are some indications that this has been particularly true in the Canadian context during the COVID-19 pandemic. Canadians have been exposed to consistent messaging from traditional media and elected political elites emphasizing the importance of significant action to combat the pandemic (Merkley et al., 2020). Despite these elite cues, misperceptions seem to have rooted themselves in a sizable portion of the Canadian population (Pennycook et al., 2020). Given the importance of elite cues to attitude formation (Zaller, 1992; Lenz, 2013), how can these misperceptions be best explained? We employ survey and social media data to argue that these attitudes are partially the result of massive Canadian consumption of U.S.-based information, with those on social media being particularly exposed.

We have two principal objectives: (1) we characterize following and sharing patterns in the Canadian social media space, comparing Twitter followership and engagement of U.S.based vs. Canada-based accounts; (2) identify associations between U.S. account followership and the spread of misinformation on Twitter, as well as between U.S. news exposure and COVID-19 misperceptions in the mass public. In doing so, we identify an important infodemic pathway and highlight how studying a single country's information ecosystem requires an empirical strategy that properly accounts for the porousness of national boundaries.

\subsection{Infodemic Pathways}

An infodemic can spread through a variety of information channels. It can be shared through traditional media where journalists, politicians, or thought leaders adopt inaccurate and misleading positions (e.g., Jamison et al., 2020). It can also be spread via peer-to-peer transmission, with rumors and unfounded conspiracies often accompanying pandemics (e.g., for HIV and AIDS see Smith et al., 1999) and issues of health more generally (e.g., on vaccines Larson, 2020). However, increased visibility into peer-to-peer interactions on social media has led to an explosion of research on how misinformation is generated and spreads on these platforms. Health information appears uniquely vulnerable to broader trends in misinformation (Krishna and Thompson, 2019), with medical informationseeking from non-official sources very common (e.g., Guess et al., 2020). Moreover, misleading medical information has been extensively documented on social media platforms, notably related to vaccines (Radzikowski et al., 2016), Ebola (Fung et al., 2016), Zika (Sharma et al., 2017), and COVID-19 (Bridgman et al., 2020). There is also evidence that false news generally spreads faster than factual news, in part due to its novelty and its capacity to generate emotive responses (Vosoughi et al., 2018).

While disinformation campaigns intended to sow confusion and uncertainty regarding COVID-19 have been documented (Swan, 2020), the infodemic appears to be sustained by a broader set of online political participants who propagate misinformation inadvertently. This "paradox of participation" is well-documented; those who are politically active online share information and can produce peer-to-peer misinformation transmission, with enthusiastic political participants being the most likely to share misinformation (Valenzuela et al., 2019). This tendency is compounded by users often adding misleading commentary and/or misrepresenting the facts as reported in the news (Anspach and Carlson, 2020). This fuels a second dynamic wherein social media users are more likely to further propagate information from those that they trust (Buchanan and Benson, 2019), more likely to believe in its veracity (Sterrett et al., 2019), more likely attribute importance to the issue (Feezell, 2018), and more likely to later trust the source (i.e., external website) of the information (Turcotte et al., 2015). Content that is widely endorsed (i.e., through likes, retweets, or shares) is more likely to be trusted still (Luo et al., 2020) through a "bandwagon heuristic" (Sundar, 2008). These effects are further compounded by an "illusory truth effect" wherein individuals have greater confidence in the truthfulness of a claim given past exposurea dynamic observed even in evaluations of fake news (Pennycook et al., 2018). Together, these psychological and behavioral phenomena can produce trust in websites and information where more caution is warranted. Ultimately, these dynamics can contribute to an infodemic and widespread misperceptions.

\subsection{U.S. Information Influence Over Canada}

The Canada-U.S. relationship has been described by former Prime Minister Pierre Trudeau as "sleeping with an elephant ...one is affected by every twitch and grunt." This continues to ring true more than 50 years after it was first uttered, with Canadian culture and media being heavily influenced by the elephantine United States media market. There is a long history of theorizing this relationship and speculating on its importance (e.g., Grant, 1965; Beattie, 1967; Barnett and McPhail, 1980; Collins, 1990; Nguyên-Duy, 1995). And indeed, fear of U.S. cultural influence has been a linchpin of a wide variety of public policies in Canada designed to protect Canadian media and culture, such as: 1) the creation of the National Film Board and the Canadian Radio Broadcasting Corporation (later the $\mathrm{CBC}$ ); and 2) Canadian content regulation by the Canadian Radiotelevision and Telecommunications Commission that requires radio and television broadcasters to air a certain share of content that is partly written, produced or contributed to by Canadians (Salter and Odartey-Wellington, 2008).

In part due to this range of government interventions, there continues to be high levels of consumption of domestic news sources for print, radio, and television media (Owen et al., 2020). Many Canadians, however, consume U.S.-based entertainment media and read, watch, or listen to U.S. news media. Unfortunately, empirical research identifying effects of U.S. information streams on the Canadian mass public has been limited (but see Trussler, 2018).

Canadians are heavy users of social media platforms, with approximately 5 in 6 having a Facebook account, 1 in 2 having an Instagram account, 2 in 5 having a Twitter account, and high usage of YouTube, LinkedIn, Pinterest, Snapchat, messaging apps, etc. (Gruzd and Mai, 2020). Borders between countries likely mean less on these platforms, with peer-to-peer networks quickly traversing national boundaries as people find 
like-minded individuals around the world. Given the shared language, special cultural relationship, and significant population imbalance, we expect Canadians to follow more U.S.-based accounts and engage more with the content produced by these accounts than Canada-based accounts.

We are interested in descriptively measuring U.S. influence on Canadian social media space and how this may vary depending on the content in question. The potential influence of U.S.based information presents a unique Canadian vulnerability during a pandemic, where Canadian elites, medical professionals, scientists, and journalists may be comparatively less able to reach and inform Canadian residents given the noise generated by U.S.-based information. Equally importantly, any information circulating in the U.S. will also impact Canadians through social media channels.

We do not have strong expectations about whether domestic Canadian COVID-19 information is more or less likely to be propagated by Canadians. On one hand, the pandemic in the United States has been more severe than in Canada which, given strong preferences for negative news consumption and sharing (Bachleda et al., 2020), may mean that Canadians have spent even more time sharing information from the U.S. On the other hand, Canadians may be more likely to focus on how COVID-19 is spreading in their communities and thus prefer local information (something that has been documented more generally on social media, e.g., Al-Rawi, 2017). Legitimate information about COVID-19 also shares space with misinformation in social media spaces, and has been often promoted by right-wing news outlets in the U.S. (Motta et al., 2020). Social media can serve as a gateway to access this information. This discussion leads us to two research questions:

RQ1: Do Canadians engage with U.S.-based information more than Canadian-based information?

RQ2: How do these patterns vary by content type (i.e., all content, COVID-19 information, and COVID-19 misinformation)?

\subsection{The U.S. as a Super-Spreader of COVID-19 Misinformation}

The second objective of this paper is to identify a possible relationship between U.S. information exposure and COVID19 misinformation or misperceptions. As noted, it is difficult for Canadians to escape the influence of U.S. media. We have reason to expect that the heavy influence of U.S.-based information has resulted in the cross-border spread of COVID19 misinformation, particularly in social media spaces.

Canada has seen a remarkable degree of elite consensus on the severity of the COVID-19 pandemic and Canadian political elites have not been important sources of COVID-19-related misinformation (Merkley et al., 2020). The political climate in the U.S. is vastly different. We see a high degree of elite polarization on the severity of COVID-19 (Green et al., 2020); this has trickled down into public opinion, with important partisan gaps emerging between Democrats and Republicans in COVID-19 risk perceptions, social distancing practice, and mask usage
(Allcott et al., 2020; Clinton et al., 2020; Kushner Gadarian et al., 2020).

Further, COVID-19 misinformation has spread well-beyond the confines of social media and alternative news websites. It is being reinforced by American media and political figures. Right-wing news outlets are propagators of COVID-19 misinformation (Motta et al., 2020), as are Republican political elites (Uscinski et al., 2020). Misinformation communicated by elite sources has the potential to reverberate throughout the entire information ecosystem; it is not just consumers of partisan media who will encounter these messages. Many Americans will be incidentally exposed to misinformation from partisan media (Lelkes et al., 2017), while mainstream sources may carry elite-sponsored misinformation to the mass public in their normal coverage of elite debate. It is not as easy for journalists to ignore communication from the President and his allies as compared to random, often times anonymous, profiles on Twitter.

Moreover, journalists may face incentives to give elite voices considerable air time. They may elevate messages featuring misinformation in a misguided attempt to maintain "balance" (Merkley, 2020). And perhaps most importantly, mainstream news outlets have increasingly found themselves in competition with partisan news outlets for viewers and readers in the U.S. This creates heightened incentives to carry polarizing messages from extreme sources (Wagner and Gruszczynski, 2018; Padgett et al., 2019). In short, American political discourse is saturated with COVID-19 misinformation to a far greater extent than peer countries, and we anticipate that this will spill over into the Canadian social media space. Consequently, U.S.-based information exposure is likely to be associated with more engagement with misinformation on social media and misperceptions related to COVID-19 in the mass public, which motivates our first hypothesis:

H1: Exposure to U.S. based information is associated with more
engagement with COVID-19 misinformation on social media (A)
and with COVID-19 misperceptions in the mass public (B).

It is also possible that U.S. news exposure conditions the relationship between social media usage and misperceptions. Social media usage has been linked to COVID-19 misperceptions because of the proliferation of related misinformation on these networks (Bridgman et al., 2020). Social media also facilitates access to U.S. information streams by breaking down national boundaries in peer-to-peer transmission. Given that these U.S. information streams are comparatively more saturated with elitesponsored misinformation, social media users with a preference for U.S. news content are more likely to be exposed to COVID-19 misinformation than those without such a preference. We anticipate important downstream implications for their (mis)perceptions related to COVID-19.

$\mathrm{H} 2:$ The association between social media usage and misperceptions is stronger among those with a preference for U.S. news. 


\section{MATERIALS AND METHODS}

To assess potential infodemic pathways into Canada, we examine exposure to, and preferences for, non-Canadian information in traditional and social media among survey respondents alongside descriptive statistics of a massive dataset of Canadian Twitter users. These two methodological approaches provide visibility into infodemic dynamics that a single method could not. They also address common concerns with self-reported information consumption (e.g., Guess, 2015) as well as differing dynamics across social media platforms (e.g., Yarchi et al., 2020).

\subsection{Social Media Data}

We use a massive multi-faceted Twitter dataset that captures actual behavior: tweets, retweets, and follow behavior of approximately 200,000 Canadian Twitter users active in $2020^{1,2}$.

We began with a labeled set of Canadian politician, journalists, and media organizations from across the media landscape in Canada ${ }^{3}$. We collected their follower network (both those they followed and those that followed them) which yielded 6,569,634 distinct users. We pulled the location and biography information for all these users. Every character sequence that appeared 10 or more times in the location fields was put through the GoogleMaps API with country data extracted to identify Canadian locations (e.g., University of Toronto is correctly placed in Toronto, Ontario). This 2-step process yielded 747,158 self-identified Canadian-based accounts. We began collecting the tweets from this population in January 2020. In September 2020, we computed the most active 200,000 accounts as measured by tweeting volume for the period from January 1 to July 31 , 2020. We then collected every account that these Canadian users followed ${ }^{4}$ yielding 9,118,496 unique users who were followed by at least one of the Canadian accounts. We collected the location of those users and ran the same location-identifying process described above. This allows us to construct an intra-country follower and retweet network across a massive set of active self-identifying Canadian Twitter accounts. Figure 1 shows the Twitter data collection process.

We examine descriptive statistics on follow and tweeting behavior for the large Canadian Twitter population. Following someone on Twitter means that a user is more likely to see their content and is taken to be a signal of interests and preferences (Barberá, 2015); it is also a commonly-used measure of exposure to information (e.g., Bail et al., 2018). Retweets are the re-sharing of information from another user to your followers and has been

\footnotetext{
${ }^{1}$ Twitter is the only social media platform where location information at the account level is readily available and where networks can be mapped. Whether the patterns identified here exist on other social media platforms is an important and unaddressed research question that cannot be effectively answered with current data access granted to academic researchers.

${ }^{2}$ All data was collected using the Twitter Standard API v1.1 as documented at: https://developer.twitter.com/en/docs/twitter-api/v1 (accessed February 16, 2021). ${ }^{3}$ Specifically, this included every Member of Parliament and every Senator with a Twitter account alongside a list of 799 journalist and media Twitter accounts manually compiled by research assistants to be as comprehensive and representative as possible.

${ }^{4}$ The follow relationships for the users were collected in October 2020 and 5,641 accounts were no longer active.
}

found to generally indicate interest, trust, and agreement with the contents of the original tweet (Metaxas et al., 2015). Tweets themselves are the precise words of the user and thus give the best indication of their attitudes and beliefs.

To identify tweets and retweets for both all COVID-19 content and COVID-19 misinformation retweets we draw upon an English-language dictionary developed by Evanega et al. $(2020)^{5,6}$. COVID-19 tweets are identified by strings "covid," "coronavirus," and "pandemic," while misinformation-associated tweets are identified by strings like: "plandemic," and "bioweapon." A dictionary approach does not allow us to identify whether a particular tweet is propagating or debunking misinformation, however it does allow us to detect tweets concerning the misinformation conversation more broadly with reasonable accuracy. We run the dictionary over the corpus of 70,996,766 geolocated retweets, yielding a total of 5,911,452 COVID-19 related retweets, of which 239,422 retweets contain terms associated with misinformation. We also run the COVID-19 and misinformation dictionaries over all $146,631,572$ tweets posted from the Canadian sample and identify 154,179 that contain terms associated with misinformation.

\subsection{Survey Data}

In addition to our social media data, we use surveys to characterize the relationship between U.S. news exposure and COVID-19 misperceptions. Our survey data come from the Media Ecosystem Observatory, which has been surveying Canadians on their COVID-19 attitudes and behaviors continuously since March 2020. In waves 9 through 15 of the survey, fielded from June 15 to August 9, $2020(N=17,331)$, a question was asked related to U.S. news media consumption. Each survey wave has an approximate sample size of 2,500 with quotas set on gender, age, Canadian region (i.e., Atlantic, Quebec, Ontario, West) and language (i.e., French, English) to match population benchmarks from the 2016 Canadian census. We further weight our data within region by age and gender using an iterative proportional fitting algorithm. More details on sample characteristics can be found in Supplementary Table 1.

\subsection{Survey Variables}

We ask our respondents "Over the past week, which of the following news media outlets did you watch, read, or listen to for news about politics? Please select all that apply." Sources

\footnotetext{
${ }^{5}$ The Canadian Twitter conversation occurs in both English and French. We expect French-language users to be less likely to follow and propagate U.S.-based information, so have excluded them from the analysis. Survey results include both English and French-speaking Canadians.

${ }^{6}$ Dictionary-based approaches are comprehensible and straightforward methods by which text can be classified. Classifying text using a dictionary-based approach is more efficient than manual annotation and can be used for large volumes of text, but the creation of the keyword list remains a subjective step in the process (Guo et al., 2016). We have amended the dictionary developed by Evanega et al. (2020) based on human annotation of a sample of tweets to ensure the list is representative of our dataset. We validated this amended dictionary through manual coding of a random selection of 500 tweets that the dictionary classified as being about misinformation, with $44 \%$ being explicitly misinformation, $39 \%$ being about misinformation (including debunking it), and only $17 \%$ not concerning misinformation at all. See Supplementary Material for the full dictionary and additional dictionary-related analyses.
} 


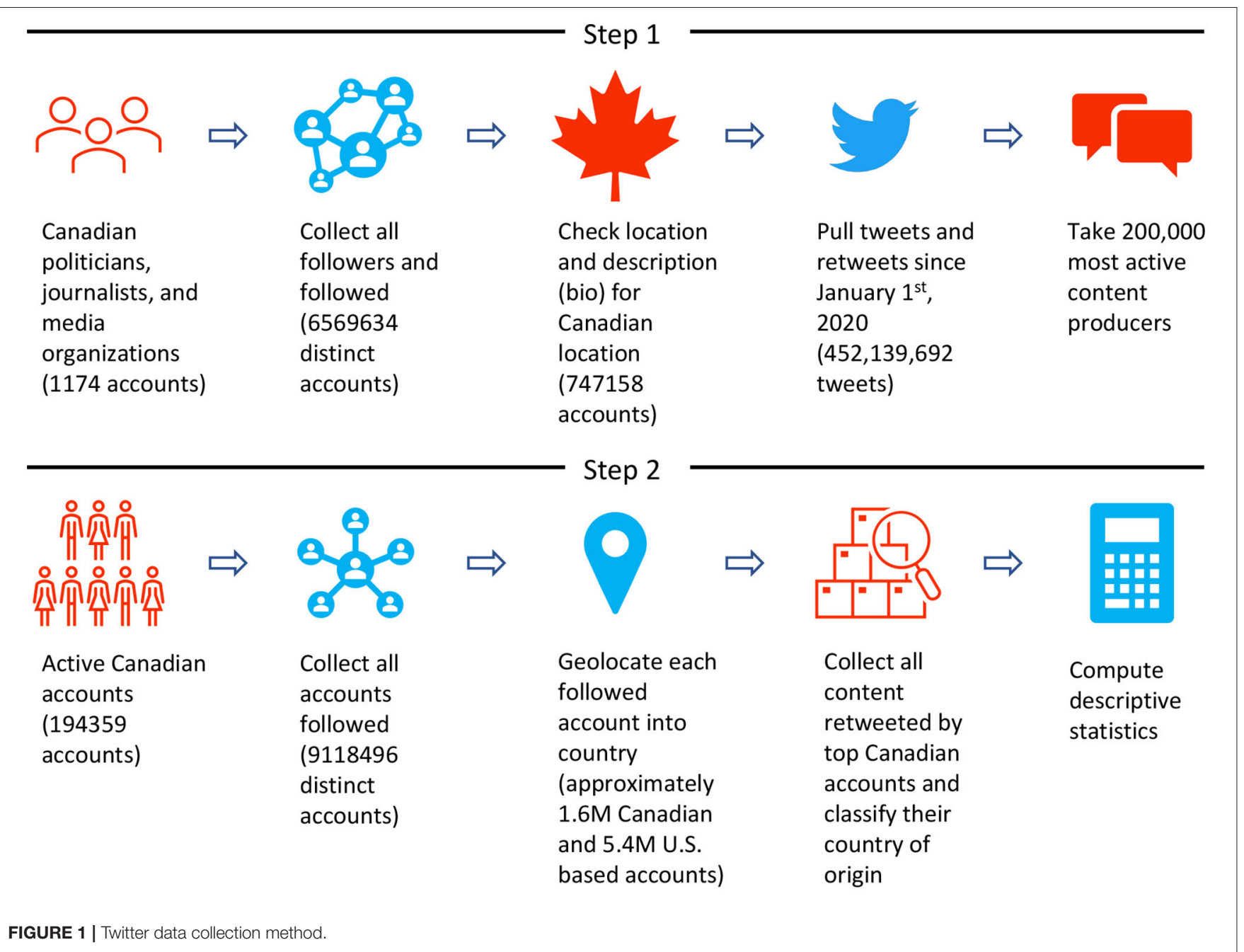

include the New York Times, Washington Post, Wall Street Journal, ABC, NBC, CBS, Fox News, CNN, MSNBC, Brietbart News, Daily Kos, NPR, Politico, The Atlantic, Bloomberg, and respondents were given the option to select none of the above. We ask a similar question for Canadian news outlets. As Table $\mathbf{1}$ shows, American news outlets command respectable audiences from the Canadian public, though domestic sources are still preferred. We construct indices of U.S. and domestic news consumption by summing the outlets selected and taking the log to account for extreme values. Results of the following analysis are robust to constructing a 4-category binned ordinal measure of U.S. news exposure, the results of which are displayed in Supplementary Table 3.

We evaluate social media usage by asking respondents the following question: "Over the past week, which of the follow social media applications did you use to watch, read, or listen to news about politics? Please select all that apply." Respondents could indicate their usage of Facebook, Twitter, Instagram, YouTube, Reddit, LinkedIn, Tumblr, Whatsapp, Snapchat, WeChat, other, or none of the above. We construct an index of social media exposure by summing the applications selected and taking the log to account for extreme values. Our media items are rescaled to $0-1$ from the minimum to the maximum. Results of the following analysis are robust to constructing a 4-category binned ordinal measure of social media exposure as well as an item asking respondents how frequently they used social media over the past week (response categories: several times a day, every day, almost every day, a few times, once, never). The results of these robustness tests are displayed in Supplementary Tables 3, 4.

We measure COVID-19 misperceptions by asking respondents to rate the following claims as definitely false, probably false, uncertain, probably true, or definitely true:

1. The coronavirus is no worse than the seasonal flu;

2. Drinking water every $15 \mathrm{~min}$ will help prevent the coronavirus;

3. The Chinese government developed the coronavirus as a bioweapon;

4. Homeopathy and home remedies can help manage and prevent the coronavirus;

5. The coronavirus was caused by the consumption of bats in China; 
TABLE 1 | U.S. and Domestic News Sources, with percentage of English Canadian respondents shown.

\begin{tabular}{llll}
\hline \multicolumn{1}{c}{ U.S. sources } & \multicolumn{2}{c}{ Domestic Canadian sources } \\
\hline CNN & 33.2 & CBC & 52.2 \\
New York Times & 15.7 & CTV & 52.0 \\
CBS & 13.7 & Global News & 42.3 \\
NBC & 12.7 & CityNews & 20.2 \\
Washington Post & 11.7 & Globe and Mail & 14.6 \\
Fox News & 11.3 & Toronto Star & 12.4 \\
ABC & 10.2 & National Post & 11.7 \\
MSNBC & 7.4 & Toronto Sun & 6.2 \\
Bloomberg & 6.6 & Rebel Media & 2.5 \\
Wall Street Journal & 5.6 & True North News & 1.7 \\
Atlantic & 3.3 & APTN & 1.6 \\
Politico & 2.3 & The Tyee & 1.2 \\
NPR & 2.3 & Post-Milennial & 1.1 \\
Breitbart & 1.2 & Press Progress & 1.0 \\
Daily Kos & 0.5 & National Observer & 1.0 \\
& & Other & 10.1 \\
None of the above & 41.3 & None of the above & 13.3 \\
\hline
\end{tabular}

6. Vitamin C can ward off the coronavirus;

7. There is a vaccine for the coronavirus that national governments and pharmaceutical companies won't release;

8. High temperatures, such as from saunas and hair dryers, can kill the coronavirus.

These items are then used to construct a $0-1$ index of misperceptions.

\subsection{Survey Models}

Our first expectation is that U.S. news exposure is associated with COVID-19 misperceptions because of the proliferation of elite-led misinformation in that national context. We test this expectation by estimating a model using Ordinary Least Squares (OLS) regression where we regress our COVID-19 misperception index on U.S news exposure and controls (X) for domestic news exposure, political discussion, political knowledge, political interest, education, age, gender, and region. More details on our controls can be found in Supplementary Table 2:

$$
\text { misperceptions }=\alpha+\beta_{1} \text { U.S. news }+X+\epsilon
$$

Our second expectation is that an association between social media exposure and COVID-19 misperceptions should be strongest among those who consume a lot of U.S. news since, as we will show, misinformation is coming primarily from U.S. sources on social media. We estimate the following model with OLS controlling for demographics, domestic news exposure and other indicators of democratic engagement and political sophistication:

$$
\begin{aligned}
\text { misperceptions }= & \alpha+\beta_{1} \text { social media }+\beta_{2} \text { U.S. news } \\
& +\beta_{3} \text { social media } * \text { U.S. news }+X+\epsilon
\end{aligned}
$$

\section{RESULTS}

We begin by descriptively characterizing following and sharing patterns cross-nationally. We find that Canadians follow far more accounts based outside of Canada than inside of Canada. Across the 187,088 English-language Canadian accounts examined here, Canadians follow a median of 36 Canadian accounts, 88 accounts from the United States, and 25 accounts from other countries. While only approximately $20 \%$ of follows that can be geolocated were identified as Canadian, 55\% were based in the United States. Looking at the distribution of the ratio between U.S.:Canadian follows shows an even more dramatic pattern. As shown in Figure 2, 71\% of Canadians follow more Americans than Canadians on Twitter and approximately $18 \%$ of Canadians follow more than 10 times as many Americans as Canadians. Canadians are exposed to more U.S.-based information on social media as compared to domestic content.

Given this follow behavior, Canadians on Twitter are likely to be receiving a lot more U.S.-based information than Canadian information. However, do they consider this information more important or does it interest them more than Canadian content, as measured by retweet volume? Figure 3 shows, from left-toright, the proportions of Canadian follows, retweets, COVID-19 retweets, and tweets containing misinformation keywords.

In addition to Canadians following far more non-Canadian accounts, they also retweet a large volume of material from the United States. Across all geolocated retweets from Canadian accounts, a full $45 \%$ are cascading U.S.-based content (only $6.8 \%$ are Canadian-Canadian retweets). For COVID-19 related information, Canadians are more likely to retweet Canadian accounts (9.1\% of overall volume) but also more likely to do so for U.S.-based content ( $47 \%$ of overall volume). Thus, while there is some relative preference for local health information as compared to all information (a result largely driven by retweeting of political and health leadership across the country), Canadians are also deeply interested in COVID-19 information coming from the United States and are still far more likely to retweet that information. Canadians are also far more likely to retweet U.S.-based misinformation. When Canadians retweet a tweet containing misinformation or about the misinformation debate, it is from a U.S.-based account $53 \%$ of the time (Canada-based accounts represent only $7.5 \%$ of volume)-a percentage far higher than both all information and COVID-19 specific information and all content regardless of topic.

We thus answer our research questions: Canadians are far more likely to follow and engage with U.S.-accounts than those from other countries, including Canada. This is particularly true for COVID-19 misinformation, though it is slightly less apparent for COVID-19 information more generally ${ }^{7}$.

\footnotetext{
${ }^{7} \mathrm{We}$ also find that social media usage is associated with U.S. news exposure among our survey respondents. We estimate model regressing U.S. news exposure on social media usage and our controls. The estimates are provided in Supplementary Table 4. Social media provides a gateway for Canadians to access U.S. news media.
} 


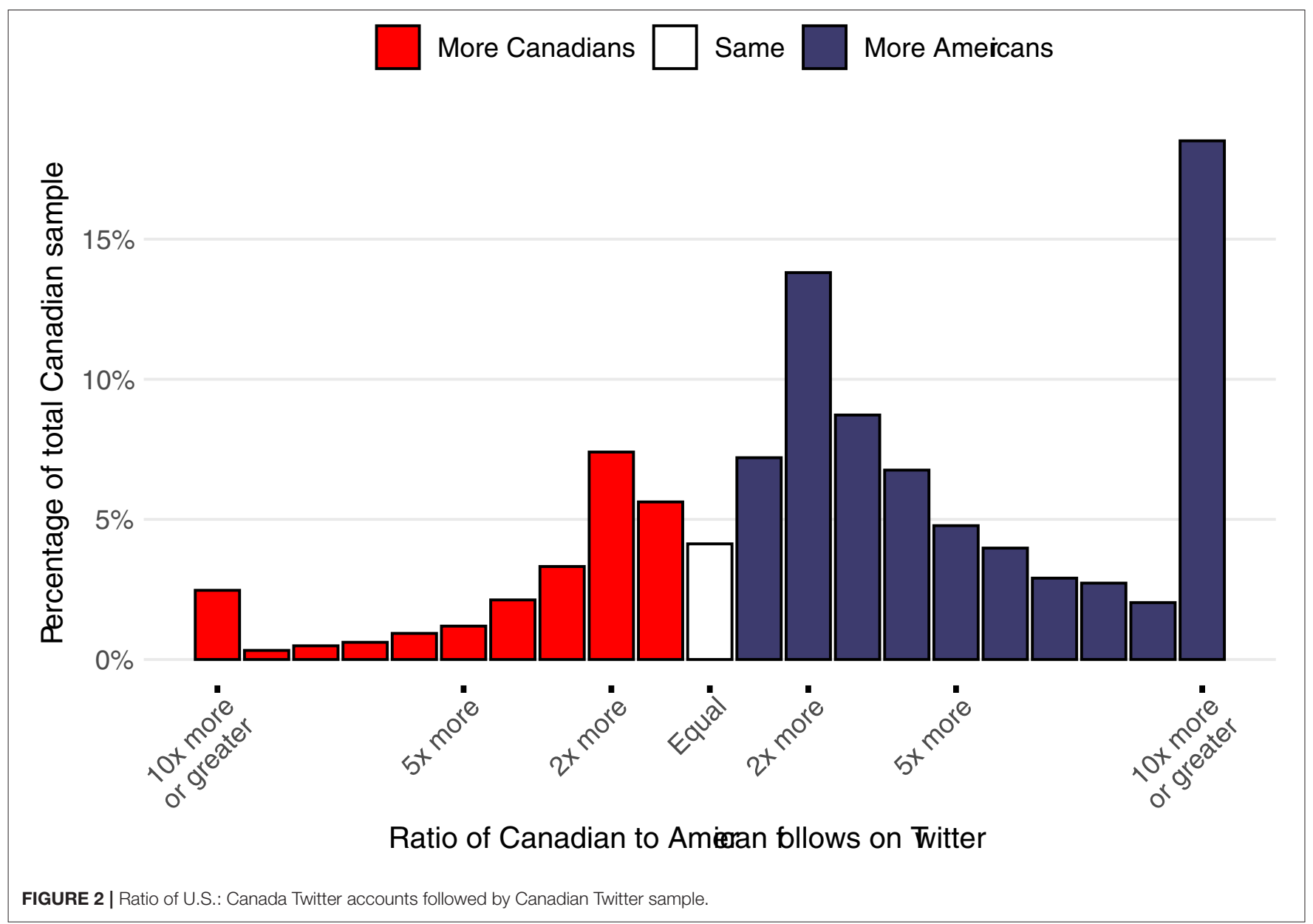

\subsection{Hypothesis Testing}

To evaluate the relationship between exposure to U.S.based information and direct propagation of COVID-19 misinformation $(H 1 A)$, we examine actual user-produced content. We calculated the percent of COVID-related tweets from each individual in the Canadian sample that contained misinformation and correlated that with the percentage of their geo-located follows that are based in the United States. We find that a 1 standard deviation increase in U.S. follows is associated with $0.061(p<0.001)$ standard deviation increase in misinformation tweeting. We find evidence for $H 1 A$ : exposure to U.S. based information is associated with more direct engagement with COVID-19 misinformation on social media.

We find that exposure to U.S. Twitter accounts is associated with posting tweets containing COVID-19 misinformation. There may be downstream effects of U.S. information exposure on misperceptions related to COVID-19 (H1B). The results of Equation (1) are presented in column 1 of Table 2. An individual with maximum level of U.S. news exposure is expected to score 0.05 points higher on the $0-1$ COVID-19 misperceptions index, which amounts to 0.27 standard deviations on this measure $(p<$ $0.001)$. $H 1 B$ is supported. U.S. news exposure is associated with more COVID-19 misperceptions after controlling for domestic news exposure and other indicators of political engagement.
The link between social media and misperceptions observed elsewhere (Bridgman et al., 2020) may be strongest among those with preference for U.S. news $(\mathrm{H} 2)^{8}$. We provide the model estimates for Equation (2) in column 3 of Table 2 and present the marginal effects in Figure 4. U.S. news exposure conditions the effect of social media on COVID-19 misperceptions. For those with no U.S. news exposure, the consumption of social media only increases COVID-19 misperceptions by 0.12 points on a $0-1$ scale. But among those with the highest level of U.S. news exposure, the observed effect of social media consumption increases more than 3 -fold. In short, social media exposure is related to COVID-19 misperceptions in large part because of its capacity to amplify the impact of content coming from the U.S. information environment.

\section{DISCUSSION}

Misinformation about COVID-19 has quickly traversed the globe, undermining efforts to contain the pandemic. For

${ }^{8} \mathrm{We}$ observe a similar correlation in the data used here as well. We estimate a model regressing COVID-19 misperceptions on social media exposure and our controls in Supplementary Table $\mathbf{5}$ and find a strong association between social media usage and misperceptions. 


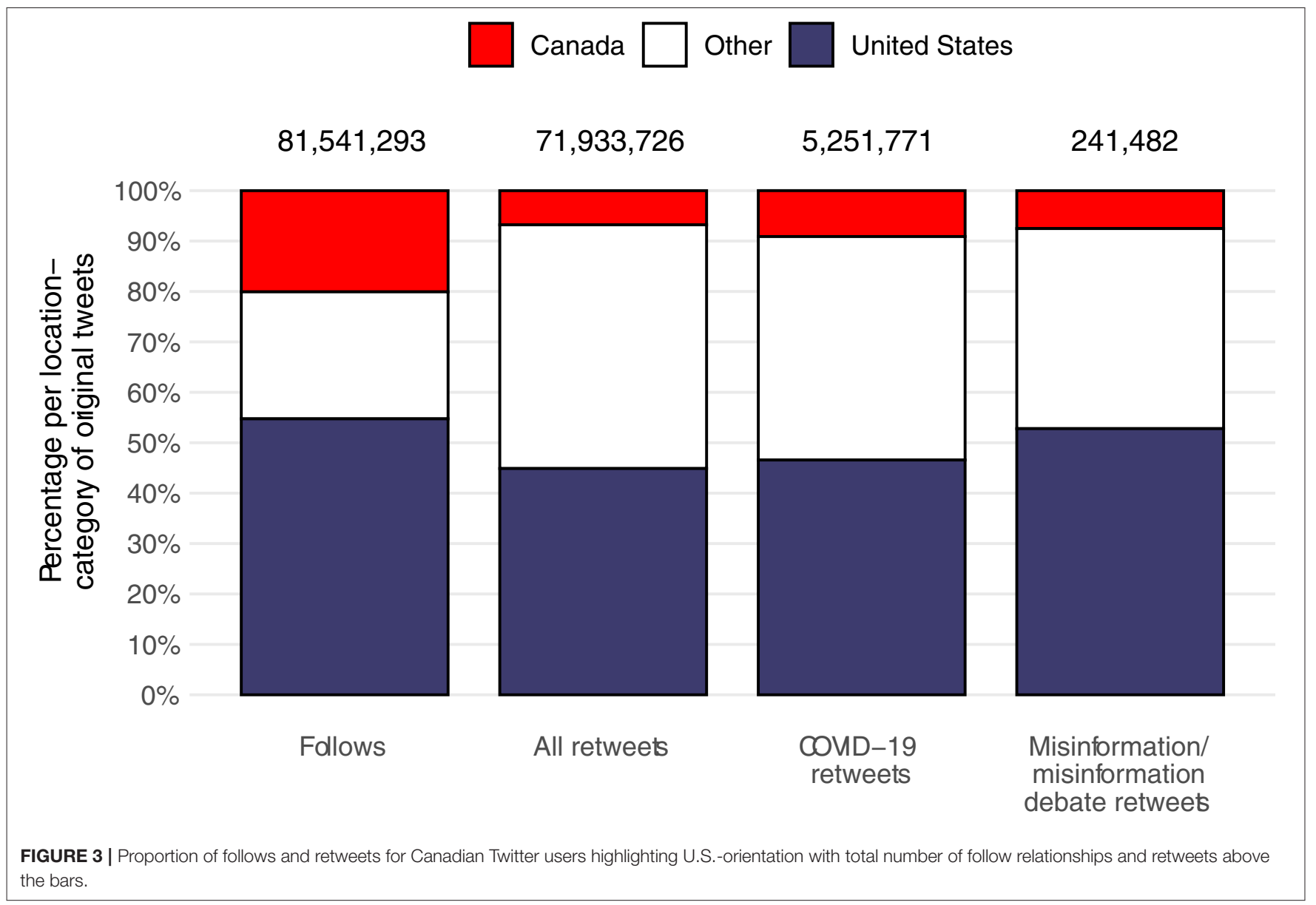

TABLE 2 | Regression estimates for survey-based models.

\begin{tabular}{|c|c|c|c|c|}
\hline & \multicolumn{2}{|c|}{ H1B } & \multicolumn{2}{|c|}{ H2 } \\
\hline & Coef. & SE & Coef. & SE \\
\hline U.S. news exposure & $0.053 * *$ & 0.008 & $-0.056 * *$ & 0.009 \\
\hline Social media exposure & & & $0.119 * *$ & 0.010 \\
\hline Social media * U.S. news & & & $0.232 * *$ & 0.027 \\
\hline Domestic news exposure & $-0.091 * *$ & 0.010 & $-0.131 * *$ & 0.010 \\
\hline Political discussion & $0.133 * *$ & 0.008 & $0.097 * *$ & 0.008 \\
\hline Political knowledge & $-0.145 * *$ & 0.006 & $-0.128 * *$ & 0.006 \\
\hline Political interest & $0.019 *$ & 0.008 & 0.004 & 0.007 \\
\hline Education & $-0.149 * *$ & 0.008 & $-0.141 * *$ & 0.008 \\
\hline Age & $-0.058 * *$ & 0.002 & $-0.037 * *$ & 0.002 \\
\hline Female & $-0.024 * *$ & 0.003 & $-0.024 * *$ & 0.003 \\
\hline Ontario & $0.029 * *$ & 0.006 & $0.028 * *$ & 0.006 \\
\hline Quebec & $0.025 * *$ & 0.006 & $0.026 * *$ & 0.006 \\
\hline West & $0.019 * *$ & 0.006 & $0.019 * *$ & 0.006 \\
\hline Constant & $0.518 * *$ & 0.009 & $0.465 * *$ & 0.010 \\
\hline $\mathrm{R}^{2}$ & \multicolumn{2}{|c|}{0.18} & \multicolumn{2}{|c|}{0.22} \\
\hline $\mathrm{N}$ & \multicolumn{2}{|c|}{16,216} & \multicolumn{2}{|c|}{16,216} \\
\hline
\end{tabular}

DV = COVID-19 misperceptions; ${ }^{*} p<0.05,{ }^{* *} p<0.01$. the Canadian case, we have shown: (1) Canadians who use social media are relatively more exposed to U.S.-based (mis)information than domestic sources of (mis)information; (2) this exposure can be linked to increased propagation of misinformation and embrace of misperceptions related to COVID-19. We have provided evidence that social media is a key conduit by which misinformation can spread cross-nationally.

These findings come with some limitations. First, we have evaluated follow and propagation patterns on Twitter as it is the only social media platform where the geographic data of users can be reasonably relied upon. We expect cross-national information transfer to be stronger on platforms with looser geographic networks (e.g., Reddit, Parler, Instagram), and weaker on platforms where geographic networks are more prominent (e.g., Facebook, Nextdoor). Existing limitations on data access make such cross-platform research difficult and more direct access to these platforms' data would be required.

Second, we have utilized a dictionary-based approach to process the enormous and diverse corpus of tweets and retweets examined here. Manual coding of the dictionary-classified content indicates that misinformation was identified alongside more general discussions of misinformation and content explicitly combating misinformation. We thus focus broadly on the misinformation conversation in this paper, but more research 


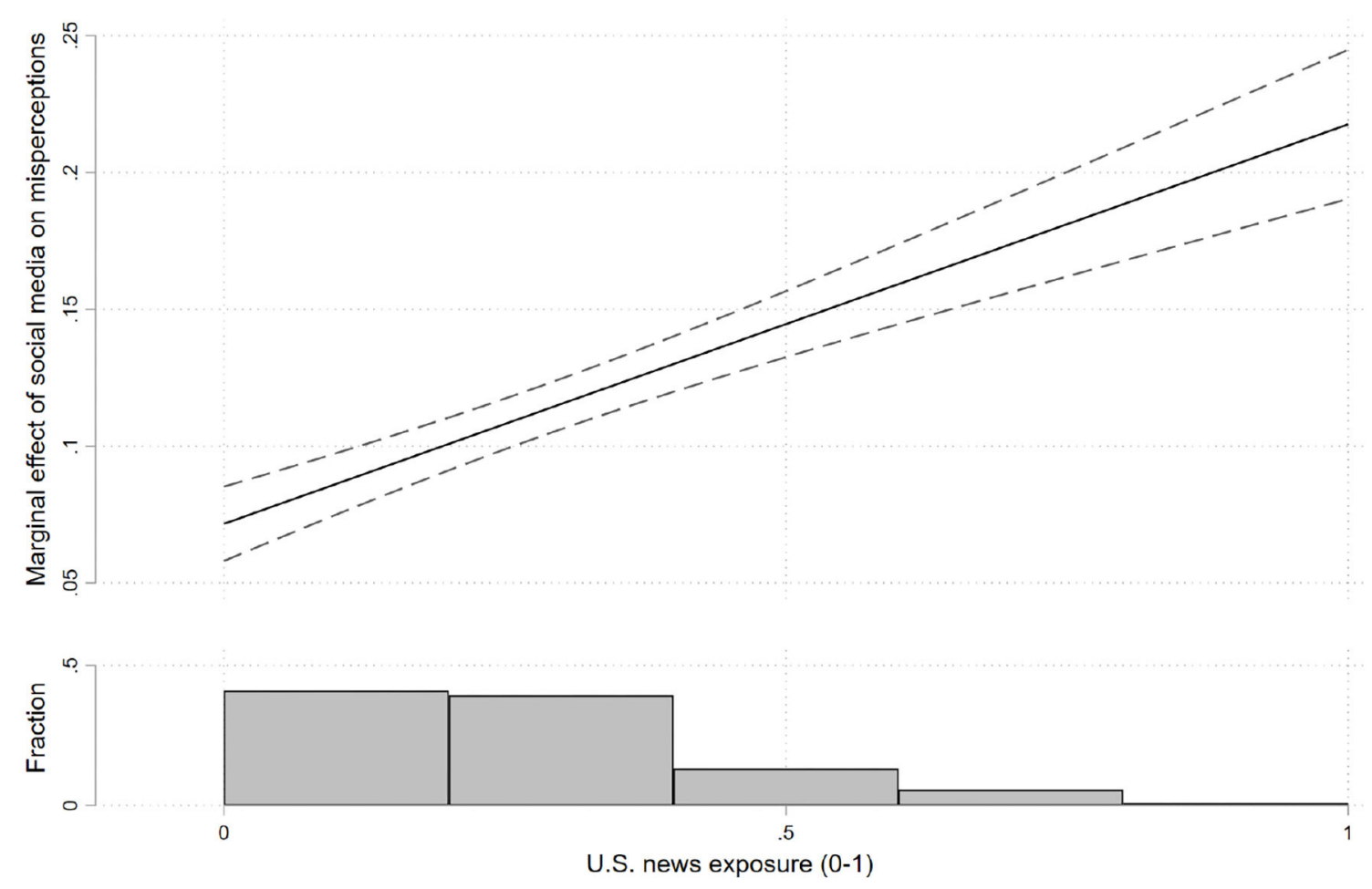

FIGURE 4 | Marginal effect of social media exposure on COVID-19 misperceptions across levels of U.S. news consumption. 95\% confidence interval.

is needed to both better identify misinformation and understand the impacts of ambiguous or anti-misinformation messages (see also Li, 2020).

Third, our data is observational and drawn from a single country case study. As such, we cannot and do not make a causal or universal argument here. However, given the enormous sample sizes and similar dynamics observed across self-reported and actual behavior, we are confident that we have accurately identified a key mechanism behind the COVID-19 infodemic in Canada. The degree to which our findings travel to other countries is likely bound by the cultural affinity, proximity, and similarity between country pairs. Canada has a uniquely close cultural relationship with the United States, with the majority of consumed popular and high culture in Canada having American origins. The degree to which such similarity and proximity is necessary to generate the information pathways described in this paper is worthy of future research.

Fourth, it must be noted that Canadians are opting into this content on social media platforms, with news consumption, follows, and retweets being active choices. However, social media platforms are likely playing a key role in deepening this exposure by saturating information streams with U.S.based news. Granting that Canadians choose to have a high interest in U.S.-based information, the content actually shown to users is algorithmically determined by social media platforms themselves. These algorithmic systems remain hidden from view, making it nearly impossible for researchers to incorporate the effects of this filtering in analyses of information exposure.
This is a broad limitation to both this study and to fully understanding and providing democratic oversight over the information ecosystem. As a result, we can't know whether the observed exposure to U.S.-based misinformation is because Canadians care deeply about all news coming from the United States or because the platform itself elevates the importance of this conversation. The latter is at least partially true and governments wishing to limit infodemic spread might consider the algorithmic ways in which social media platforms incidentally push out-of-country information to the top of news feeds.

Previous work on the information ecosystem during the Canadian election found little evidence of mis- and disinformation flowing north from the U.S. (e.g., Owen et al., 2020). We speculate that this is simply because there was not much content on the Canadian election produced in the United States. By contrast, an international event like the COVID-19 pandemic is of shared importance, with information produced in the United States of interest to news consumers in Canada. Additional research needs to be done to evaluate whether other international issues, such as immigration, refugee crises, climate change, or international relations are subject to similar dynamics. We can speculate, however, that Canadian discourse in these areas of shared interest is similarly saturated with U.S.-based (mis)information.

Our work thus has important implications for policy makers who wish to confront the deleterious effects of misinformation. Around the world, democratic governments are exploring 
legislative and regulatory solutions to limit the spread of misinformation. However, their reach does not easily extend to producers of content and users of social media platforms who exist outside of their borders. Our research shows that these out-of-country sources can be a key source and conduit of misinformation. Thus, if policy makers are looking to limit future waves of misinformation or encourage greater nationallybounded deliberation on social media, a focus on the information pathways for topics that easily transfer between countries is warranted.

\section{DATA AVAILABILITY STATEMENT}

Data and replication materials are available at https://doi.org/10. 7910/DVN/Y0RP9J.

\section{ETHICS STATEMENT}

The studies involving human participants were reviewed and approved by the University of Toronto Social Sciences, Humanities and Education REB, protocol \#00038251. The patients/participants provided their written and informed consent to participate in this study.

\section{REFERENCES}

Allcott, H., Boxell, L., Conway, J., Gentzkow, M., Thaler, M., and Yang, D. (2020). "Polarization and public health: partisan differences in social distancing during the coronavirus pandemic. J. Pub. Econ. 191:104254. doi: 10.1016/j.jpubeco.2020.104254

Al-Rawi, A. (2017). Audience preferences of news stories on social media? J. Soc. Media Soc. 6, 343-367. Available online at: https://thejsms.org/index.php/ TSMRI/article/view/284

Anspach, N. M., and Carlson, T. N. (2020). What to believe? Social media commentary and belief in misinformation. Polit. Behav. 42, 697-718. doi: 10.1007/s11109-018-9515-Z

Bachleda, S., Neuner, F., Soroka, S., Guggenheim, L., Fournier, P., and Naurin, E. (2020). Individual-level differences in negativity biases in news selection. Pers. Indiv. Differ. 155:109675. doi: 10.1016/j.paid.2019.109675

Bail, C. A., Argyle, L. P., Brown, T. W., Bumpus, J. P., Chen, H., Hunzaker, M. B. F., et al. (2018). Exposure to opposing views on social media can increase political polarization. Proc. Natl. Acad. Sci. U.S.A. 115, 9216-9221. doi: $10.1073 /$ pnas. 1804840115

Barberá, P. (2015). Birds of the same feather tweet together: bayesian ideal point estimation using twitter data. Polit. Anal. 23, 76-91. doi: 10.1093/pan/mpu011

Barnett, G., and McPhail, T. (1980). An examination of the relationship of United States television and Canadian identity. Int. J. Intercult. Relat. 4, 219-232. doi: 10.1016/0147-1767(80)90030-9

Beattie, E. (1967). In Canada's centennial year, U. S. mass media influence probed. J. Mass Commun. Q. 44, 667-672. doi: 10.1177/107769906704400406

Bridgman, A., Merkley, E., Loewen, P. J., Owen, T., Ruths, D., Teichmann, L., et al. (2020). The causes and consequences of COVID-19 misperceptions: understanding the role of news and social media. Harvard Kennedy School Misinform. Rev. 1. doi: 10.37016/mr-2020-028

Buchanan, T., and Benson, V. (2019). Spreading disinformation on facebook: do trust in message source, risk propensity, or personality affect the organic reach of "fake news"? Soc. Med. Soc. 5:2056305119888654. doi: $10.1177 / 2056305119888654$

\section{AUTHOR CONTRIBUTIONS}

$\mathrm{AB}$ and EM developed the theory, research design, hypotheses, analysis plan, and drafted the main sections of the manuscript. $\mathrm{AB}$ developed the data collection for the social media side, analyzed the data, and drafted the social media results. EM collected and analyzed the survey data and drafted those results. OZ helped with data collection and analysis for the social media data. PL, TO, and DR secured and provided funding to support the project and reviewed and edited the manuscript. All authors contributed to the discussion.

\section{FUNDING}

The project was funded in part through the Department of Canadian Heritage's Digital Citizens Initiative, the Office of the Dean of the Faculty of Arts \& Science, University of Toronto, and the Social Sciences and Humanities Research Council.

\section{SUPPLEMENTARY MATERIAL}

The Supplementary Material for this article can be found online at: https://www.frontiersin.org/articles/10.3389/fpos. 2021.648646/full\#supplementary-material

Clinton, J., Cohen, J., Lapinski, J., and Trussler, M. (2020). Partisan pandemic: how partisanship and public health concerns affect individuals' social mobility during COVID-19. Sci. Adv. 7:eabd7204. doi: 10.2139/ssrn.3633934

Collins, R. (1990). Television: Policy and Culture. London: Taylor \& Francis.

Evanega, S., Lynas, M., Adams, J., and Smolenyak, K. (2020). Coronavirus misinformation: quantifying sources and themes in the COVID-19 'infodemic'. doi: 10.2196/preprints. 25143

Feezell, J. T. (2018). Agenda setting through social media: the importance of incidental news exposure and social filtering in the digital era. Polit. Res. Q. 71, 482-494. doi: 10.1177/1065912917744895

Fung, I. C. H., Fu, K. W., Chan, C. H., Chan, B. S. B., Cheung, C. N., Abraham, T., et al. (2016). Social media's initial reaction to information and misinformation on Ebola, August 2014: facts and rumors. Public Health Rep. 131, 461-473. doi: $10.1177 / 003335491613100312$

Grant, G. (1965). Lament for a Nation: The Defeat of Canadian Nationalism. Montréal: McGill-Queen's Press.

Green, J., Edgerton, J., Naftel, D., Shoub, K., and Cranmer, S. J. (2020). Elusive consensus: polarization in elite communication on the COVID-19 pandemic. Sci. Adv. 6:eabc2717. doi: 10.1126/sciadv.abc2717

Gruzd, A., and Mai, P. (2020). The State of Social Media in Canada 2020. SSRN Scholarly Paper ID 3651206. Rochester, NY: Social Science Research Network.

Guess, A. M. (2015). Measure for measure: an experimental test of online political media exposure. Polit. Anal. 23, 59-75. doi: 10.1093/pan/mpu010

Guess, A. M., Nyhan, B., O'Keeffe, Z., and Reifler, J. (2020). The sources and correlates of exposure to vaccine-related (mis)information online. Vaccine 38, 7799-7805. doi: 10.1016/j.vaccine.2020.10.018

Guo, L., Vargo, C. J., Pan, Z., Ding, W., and Ishwar, P. (2016). Big social data analytics in journalism and mass communication: comparing dictionary-based text analysis and unsupervised topic modeling. J. Mass Commun. Q. 93, 332-359. doi: 10.1177/1077699016639231

Jamison, A. M., Broniatowski, D. A., Dredze, M., Sangraula, A., Smith, M. C., and Quinn, S. C. (2020). Not just conspiracy theories: vaccine opponents and proponents add to the COVID-19 'infodemic' on Twitter. Harvard Kennedy School Misinform. Rev. 1. doi: 10.37016/mr-2020-38 
Jang, J., Lee, E. J., and Shin, S. Y. (2019). What debunking of misinformation does and doesn't. Cyberpsychol. Behav. Soc. Netw. 22, 423-427. doi: $10.1089 /$ cyber.2018.0608

Kim, L., Fast, S. M., and Markuzon, N. (2019). Incorporating media data into a model of infectious disease transmission. PLOS ONE 14:e0197646. doi: 10.1371/journal.pone.0197646

Krishna, A., and Thompson, T. L. (2019). Misinformation about health: a review of health communication and misinformation scholarship. Am. Behav. Sci. 65, 316-332. doi: 10.1177/0002764219878223

Kushner Gadarian, S., Goodman, S. W., and Pepinsky, T. B. (2020). Partisanship, Health Behavior, and Policy Attitudes in the Early Stages of the COVID-19 Pandemic. SSRN Scholarly Paper ID 3562796. Rochester, NY: Social Science Research Network.

Larson, H. (2018). The biggest pandemic risk? Viral misinformation. Nature 562, 309-310. doi: 10.1038/d41586-018-07034-4

Larson, H. (2020). Stuck: How Vaccine Rumors Start-And Why They Don't Go Away. Oxford: Oxford University Press.

Lelkes, Y., Sood, G., and Iyengar, S. (2017). The hostile audience: the effect of access to broadband internet on partisan affect. Am. J. Polit. Sci. 61, 5-20. doi: 10.1111/ajps.12237

Lenz, G. S. (2013). Follow the Leader? How Voters Respond to Politicians' Policies and Performance. Chicago: University of Chicago Press.

Li, J. (2020). Toward a research agenda on political misinformation and corrective information. Polit. Commun. 37, 125-135. doi: 10.1080/10584609.2020.1716499

Luo, M., Hancock, J. T., and Markowitz, D. M. (2020). Credibility perceptions and detection accuracy of fake news headlines on social media: effects of truth-bias and endorsement cues. Commun. Res. 00, 1-25. doi: 10.1177/0093650220921321

Merkley, E. (2020). Are experts (news)worthy? Balance, conflict, and mass media coverage of expert consensus. Polit. Commun. 37, 530-549. doi: 10.1080/10584609.2020.1713269

Merkley, E., Bridgman, A., Loewen, P. J., Owen, T., Ruths, D., and Zhilin, O. (2020). A rare moment of cross-partisan consensus: elite and public response to the COVID-19 pandemic in Canada. Can. J. Polit. Sci. 53, 1-8. doi: 10.1017/S0008423920000311

Metaxas, P. T., Mustafaraj, E., Wong, K., Zeng, L., O’Keefe, M., and Finn, S. (2015). "What do retweets indicate? Results from user survey and meta-review of research," in Proc. Ninth Int. AAAI Conf. Web Soc. Med. Oxford.

Motta, M., Stecula, D., and Farhart, C. (2020). How right-leaning media coverage of COVID-19 facilitated the spread of misinformation in the early stages of the pandemic in the U.S. Cana. J. Poli. Sci. 53, 335-342. doi: 10.1017/S0008423920000396

Nguyên-Duy, V. (1995). Frontières L'influence américaine sur la télévision et la culture québécoises. Québec Français 98, 77-84.

Owen, T., Loewen, P., Ruths, D., Bridgman, A., Gorwa, R., MacLellan, S., et al. (2020). Lessons in Resilience: Canada's Digital Media Ecosystem and the 2019 Election. Technical report, Public Policy Forum.

Padgett, J., Dunaway, J. L., and Darr, J. P. (2019). As seen on TV? How gatekeeping makes the U.S. house seem more extreme. J. Commun. 69, 696-719. doi: 10.1093/joc/jqz039

Pennycook, G., Cannon, T. D., and Rand, D. G. (2018). Prior exposure increases perceived accuracy of fake news. J. Exp. Psychol. Gen. 147, 1865-1880. doi: $10.1037 /$ xge0000465

Pennycook, G., McPhetres, J., Bago, B., and Rand, D. (2020). Predictors of Attitudes and Misperceptions About COVID-19 in Canada, the U.K., and the U.S.A. OSF. doi: 10.31234/osf.io/zhjkp

Radzikowski, J., Stefanidis, A., Jacobsen, K. H., Croitoru, A., Crooks, A., and Delamater, P. L. (2016). The measles vaccination narrative in twitter: a quantitative analysis. JMIR Public Health Surveill. 2:e1. doi: 10.2196/publichealth.5059

Salter, L., and Odartey-Wellington, F. N. (2008). The CRTC and Broadcasting Regulation in Canada. Toronto: Carswell.

Sharma, M., Yadav, K., Yadav, N., and Ferdinand, K. C. (2017). Zika virus pandemic-analysis of Facebook as a social media health information platform. Am. J. Infect. Control 45, 301-302. doi: 10.1016/j.ajic.2016. 08.022

Smith, L. C., Lucas, K. J., and Latkin, C. (1999). Rumor and gossip: social discourse on HIV and AIDS. Anthropol. Med. 6, 121-131. doi: 10.1080/13648470.1999.9964577

Sterrett, D., Malato, D., Benz, J., Kantor, L., Tompson, T., Rosenstiel, T., et al. (2019). Who shared it? Deciding what news to trust on social media. Digit. J. 7, 783-801. doi: 10.1080/21670811.2019.1623702

Sundar, S. S. (2008). "The MAIN model: a heuristic approach to understanding technology effects on credibility," in Digital Media, Youth, and Credibility, eds M. J. Metzger and A. J. Flanagin (Cambridge, MA: MIT Press), 73-100.

Swan, B. W. (2020). State Report: Russian, Chinese and Iranian Disinformation Narratives Echo One Another. Virginia: Politico. Available online at: https:// www.politico.com/news/2020/04/21/russia-china-iran-disinformationcoronavirus-state-department- 193107

Trussler, M. (2018). Elephant Over the Airwaves: Examining the Influence of American Media on Canadian Elections. Presented at CPSA Annual Conference 2018 in Regina.

Turcotte, J., York, C., Irving, J., Scholl, R. M., and Pingree, R. J. (2015). News recommendations from social media opinion leaders: effects on media trust and information seeking. J. Comput. Mediat. Commun. 20, 520-535. doi: $10.1111 /$ jcc4.12127

Uscinski, J. E., Enders, A. M., Klofstad, C., Seelig, M., Funchion, J., Everett, C. et al. (2020). Why do people believe COVID-19 conspiracy theories? Harvard Kennedy School Misinform. Rev. 1. doi: 10.37016/mr-2020-015

Valenzuela, S., Halpern, D., Katz, J. E., and Miranda, J. P. (2019). The paradox of participation versus misinformation: social media, political engagement, and the spread of misinformation. Digit. J. 7, 802-823. doi: 10.1080/21670811.2019.1623701

Vosoughi, S., Roy, D., and Aral, S. (2018). The spread of true and false news online. Science 359, 1146-1151. doi: 10.1126/science.aap9559

Wagner, M. W., and Gruszczynski, M. (2018). Who gets covered? Ideological extremity and news coverage of members of the U.S. Congress, 1993 to 2013. J. Mass Commun. Q. 95, 670-690. doi: 10.1177/10776990177 02836

Yarchi, M., Baden, C., and Kligler-Vilenchik, N. (2020). Political polarization on the digital sphere: a cross-platform, over-time analysis of interactional, positional, and affective polarization on social media. Polit. Commun. 1-42. doi: 10.1080/10584609.2020.1785067

Zaller, J. R. (1992). The Nature and Origins of Mass Opinion. Cambridge: Cambridge University Press.

Conflict of Interest: The authors declare that the research was conducted in the absence of any commercial or financial relationships that could be construed as a potential conflict of interest.

Copyright (c) 2021 Bridgman, Merkley, Zhilin, Loewen, Owen and Ruths. This is an open-access article distributed under the terms of the Creative Commons Attribution License (CC BY). The use, distribution or reproduction in other forums is permitted, provided the original author(s) and the copyright owner(s) are credited and that the original publication in this journal is cited, in accordance with accepted academic practice. No use, distribution or reproduction is permitted which does not comply with these terms. 